Samir Jaber

Boris Jung

Philippe Corne

Mustapha Sebbane

Laurent Muller

Gerald Chanques

Daniel Verzilli

Olivier Jonquet

Jean-Jacques Eledjam

Jean-Yves Lefrant

\title{
An intervention to decrease complications related to endotracheal intubation in the intensive care unit: a prospective, multiple-center study
}

L. Muller $\cdot$ J.-Y. Lefrant

Fédération Anesthésie-Douleur-

Urgences-Réanimation,

Groupe Hospitalo-Universitaire Caremeau, Centre Hospitalier Universitaire Nîmes, Place du Professeur Robert Debré, 30029 Nîmes Cedex 9, France

Abstract Objective: To determined whether the implementation of an intubation management protocol leads to the reduction of intubationrelated complications in the intensive care unit (ICU). Design: Twophase, prospective, multicenter controlled study. Setting: Three medical-surgical ICUs in two university hospitals. Patients: Two hundred three consecutive ICU patients required 244 intubations. Interventions: All intubations performed during two consecutive phases (a 6-month quality control phase followed by a 6-month intervention phase based on the implementation of an ICU intubation bundle management protocol) were evaluated. The ten bundle components were: preoxygenation with noninvasive positive pressure ventilation, presence of two operators, rapid sequence induction, cricoid pressure, capnography, protective ventilation, fluid loading, preparation and early administration of sedation and vasopressor use if needed.
Measurements and main results: The primary end points were the incidence of life-threatening complications occurring within $60 \mathrm{~min}$ after intubation (cardiac arrest or death, severe cardiovascular collapse and hypoxemia). Other complications (mild to moderate) were also evaluated. Baseline characteristics, including demographic data and reason for intubation (mainly acute respiratory failure), were similar in the two phases. The intubation procedure in the intervention phase ( $n=121)$ was associated with significant decreases in both lifethreatening complications (21 vs. $34 \%, p=0.03)$ and other complications ( 9 vs. $21 \%, p=0.01$ ) compared to the control phase $(n=123)$. Conclusions: The implementation of an intubation management protocol can reduce immediate severe life-threatening complications associated with intubation of ICU patients.

Keywords Intubation - Airway management - Complications · Mechanical ventilation . Non-invasive ventilation . Practice guidelines

\author{
Service de Réanimation Médicale \\ Assistance Respiratoire, \\ CHU de Montpellier, \\ Hôpital Gui-de-Chauliac, \\ 80 avenue Augustin Fliche, \\ 34295 Montpellier Cedex 5, France \\ G. Chanques - D. Verzilli - J.-J. Eledjam \\ CHU de Montpellier, Saint Eloi Teaching \\ 80 avenue Augustin Fliche, \\ e-mail: s-jaber@chu-montpellier.fr \\ Tel.: +33-467-337271 \\ B. Jung $\cdot$ P. Corne $\cdot$ O. Jonquet

e




\section{Introduction}

Endotracheal intubation, which is one of the most commonly performed procedures in the intensive care unit (ICU) [1-4], is associated with a high incidence of complications (25 to $39 \%$ ) because of the precarious hemodynamic and respiratory status of critically ill patients $[1,2,5-8]$. In anesthesia and pre-hospital practices, specific procedures [i.e., preoxygenation, rapid sequence intubation (RSI) using the combined administration of a sedative and muscle relaxant agent, capnography to check the correct tube placement, etc.] are included in guidelines and are recommended to improve intubation safety [9-12]. Such standardized recommendations are lacking in ICU practice. Contrary to airway management performed in the operating room and pre-hospital conditions, few studies designed to improve intubation safety in the ICU have been published [13]. We recently showed that non-invasive positive pressure ventilation (NIPPV) improved preoxygenation of obese patients before intubation both in the ICU [1] and in the operating room [14].

We hypothesized that associating preoxygenation NIPPV with other procedures that have been shown to improve intubation safety according to pre-hospital and anesthesia literature may decrease the incidence of immediate complications after intubation in the ICU. Employing a group of evidence-based treatments related to a disease process, instituted together over a specific timeframe and termed "a care bundle," is expected to result in better outcomes than when the treatments are executed individually. For instance, when "the care bundle" is implemented as rapidly as possible, it improves the management of outof-hospital respiratory distress [15], sedation and weaning from the ventilator [16, 17], as well as severe sepsis [18, 19] and surgical procedures [20]. We therefore conducted a prospective before-after study to determine whether the implementation of an intubation bundle therapy results in a reduction of intubation-related complications in the ICU.

\section{Patients and methods}

The present study was performed in three ICUs. Data from all ICU tracheal intubations were collected and analyzed. We excluded those performed for cardiac arrest. The protocol was approved by the local ethics committee.

\section{Study design (see ESM) \\ Control phase}

During a 6-month control phase, intubation procedures were performed without the use of a protocol by the clinicians in charge of the patient.

\section{Interphase}

Prior to beginning enrollment in the intervention phase, we developed an intubation care bundle management system based on a review of the ICU airway literature $[1,2,4,9,11,16,21,22]$ (Table 1). During a 4-week phase, all physicians, residents and nurses received formal education and clinical training for the processes and procedures related to the ten-point bundle management.

\section{Intervention phase}

During the 6-month intervention phase, the recommended intubation procedure was to conform to the bundle management detailed in Table 1 (see ESM).

\section{Measurements and definitions of complications}

We documented baseline characteristics, reason for admission to ICU and status of the following before intubation: reason for intubation, heart rate, systolic arterial blood pressure, use of vasopressive drugs and pulse oxygen saturation level $\left(\mathrm{SpO}_{2}\right)$. During the intubation procedure, we documented drug administration and the operator status (airway expert vs. non expert physician) [7].

We then recorded complications and categorized as severe life-threatening or mild to moderate complications (see ESM and Table 2) that occurred during the intubation

Table 1 Intubation care bundle management

\section{Pre-intubation}

1. Presence of two operators

2. Fluid loading (isotonic saline $500 \mathrm{ml}$ or starch $250 \mathrm{ml}$ ) in absence of cardiogenic pulmonary edema

3. Preparation of long-term sedation

4. Preoxygenation for $3 \mathrm{~min}$ with NIPPV in case of acute respiratory failure $\left(\mathrm{FiO}_{2} 100 \%\right.$, pressure support ventilation level between 5 and $15 \mathrm{cmH}_{2} \mathrm{O}$ to obtain an expiratory tidal volume between 6 and $8 \mathrm{ml} / \mathrm{kg}$ and PEEP of $5 \mathrm{cmH}_{2} \mathrm{O}$ ) During intubation

5 . Rapid sequence induction: etomidate $0.2-0.3 \mathrm{mg} / \mathrm{kg}$ or ketamine $1.5-3 \mathrm{mg} / \mathrm{kg}$ combined with succinylcholine $1-1.5 \mathrm{mg} / \mathrm{kg}$ in absence of allergy, hyperkaliemia, severe acidosis, acute or chronic neuromuscular disease, burn patient for more than $48 \mathrm{~h}$ and medullar trauma

6. Sellick maneuver

Post-intubation

7. Immediate confirmation of tube placement by capnography

8. Norepinephrine if diastolic blood pressure remains $<35 \mathrm{mmHg}$

9. Initiate long-term sedation

10. Initial "protective ventilation": tidal volume $6-8 \mathrm{ml} / \mathrm{kg}$ of ideal body weight, $\mathrm{PEEP}<5 \mathrm{cmH}_{2} \mathrm{O}$ and respiratory rate between 10 and 20 cycles/min, $\mathrm{FiO}_{2} 100 \%$ for a plateau pressure $<30 \mathrm{cmH}_{2} \mathrm{O}$

NIPPV non-invasive positive pressure ventilation, $P E E P$ positive end expiratory pressure, $\mathrm{FiO}_{2}$ inspired oxygen fraction 
Table 2 Definitions of severe life-threatening and mild to moderate complications related to intubation procedure

Severe life-threatening

Death

Cardiac arrest

Severe cardiovascular collapse defined as systolic blood pressure $<65 \mathrm{mmHg}$ recorded at least one time and/or $<90 \mathrm{mmHg}$ that lasted 30-min despite $500-1,000 \mathrm{ml}$ of fluid loading (crystalloids/or colloids solutions) and/or requiring introduction of vasoactive support

Severe hypoxemia (decrease in $\mathrm{SpO}_{2}$ level to less than $80 \%$ during attempts)

Mild to moderate complications

Difficult intubation (three or more attempts at laryngoscopy to place the endotracheal tube into the trachea and/or $>10 \mathrm{~min}$ using conventional laryngoscopy and/or the need for another operator)

Esophageal intubation

Aspiration of gastric contents (migration of stomach contents into the lung)

Supraventricular and/or ventricular arrhythmia (without pulseless rhythm) that required therapy

Dangerous agitation (Richmond Agitation-Sedation Scale score above 3)

Dental injury

and within the first hour after intubation. Outcome was evaluated by length of mechanical ventilation, number of ventilator-free days during ICU stay, length of stay in the ICU and vital status upon ICU discharge.

\section{Statistical analysis}

Data are expressed as mean $\pm \mathrm{SD}$, median [25-75th] for non-Gaussian variables, or number and percentage with 95\% confidence interval. Comparison of two proportions was performed with use of the chi-square test or Fischer exact test where appropriate, comparison of means was performed with use of the Student's $t$ test, and comparison of medians was performed with the Mann-Whitney test. A $p$ value $<0.05$ was considered significant.

\section{Results (see ESM)}

\section{Population}

We compared 121 intubations in the control group to 123 in the intervention group. The two groups were similar at baseline (Table 3). Variables obtained before intubation are reported in Table 4.

Intubation procedure description

The processes of medical care for the intubation procedures are reported in Table 5. In the interventional group, in
Table 3 Baseline characteristics of the 203 studied patients

\begin{tabular}{llll}
\hline & $\begin{array}{l}\text { Control } \\
(n=101)\end{array}$ & $\begin{array}{l}\text { Intervention } \\
(n=102)\end{array}$ & $p$ \\
\hline Age (years) & $64 \pm 15$ & $61 \pm 17$ & 0.93 \\
Male gender & $67(66)$ & $66(65)$ & 0.99 \\
SAPS II & $47 \pm 18$ & $46 \pm 19$ & 0.58 \\
Weight (kg) & $74 \pm 20$ & $74 \pm 24$ & 0.97 \\
Body mass index $\left(\mathrm{kg} \mathrm{m}^{-2}\right)$ & $26 \pm 6$ & $26 \pm 8$ & 0.84 \\
Type of admission & $76(75)$ & $73(72)$ & 0.66 \\
$\quad$ Medical & $35(25)$ & $29(28)$ & 0.42 \\
$\quad$ Surgical & & & \\
Reason for ICU admission & & & \\
$\quad$ Acute respiratory failure & $43(42)$ & $42(41)$ & 0.95 \\
Shock & $15(15)$ & $18(18)$ & 0.73 \\
Trauma & $1(1)$ & $3(3)$ & 0.62 \\
Postoperative & $10(10)$ & $12(12)$ & 0.84 \\
Cardiac arrest & $3(3)$ & $1(1)$ & 0.61 \\
Neurologic & $14(14)$ & $12(12)$ & 0.81 \\
Others & $15(15)$ & $14(13)$ & 0.98 \\
\hline
\end{tabular}

Data are number (\%) or mean (SD). ICU Intensive Care Unit, SAPS II Simplified Acute Physiologic Score [36]

No significant differences were observed

Table 4 Operator status and main variables of the 244 evaluated procedures obtained before intubation

\begin{tabular}{llll}
\hline & $\begin{array}{l}\text { Control } \\
(n=121)\end{array}$ & $\begin{array}{l}\text { Intervention } \\
(n=123)\end{array}$ & $p$ \\
\hline Reason for intubation & & & \\
$\quad$ Acute respiratory failure & $73(60)$ & $80(65)$ & 0.33 \\
Shock & $13(11)$ & $16(13)$ & 0.71 \\
Coma & $15(12)$ & $14(11)$ & 0.98 \\
Replace the endotracheal tube & $5(4)$ & $1(1)$ & 0.21 \\
Unplanned extubation & $7(6)$ & $4(3)$ & 0.52 \\
Others & $8(7)$ & $8(7)$ & $>0.99$ \\
Time & $58(48)$ & $67(55)$ & 0.29 \\
Day & $63(52)$ & $56(45)$ & 0.35 \\
Night & $89(72)$ & $99(82)$ & 0.03 \\
Expert operator & $69(57)$ & $73(60)$ & 0.72 \\
Informed patient & $102 \pm 33$ & $108 \pm 30$ & 0.17 \\
Lowest systolic arterial & & & \\
$\quad$ blood pressure (mmHg) & $87 \pm 10$ & $86 \pm 16$ & 0.44 \\
SpO ${ }_{2}$ (\%) & & \\
Emergency characteristic of intubation & $45(38)$ & $44(36)$ & 0.95 \\
Real emergency & $57(47)$ & $59(49)$ & 0.95 \\
Relative emergency & $19(16)$ & $18(15)$ & 0.53 \\
Deferred emergency & $21(18)$ & $19(16)$ & 0.83 \\
Vasopressors use & $11 \pm 4$ & $12 \pm 4$ & 0.55 \\
Glasgow Coma Scale &
\end{tabular}

The emergency characteristic of intubation was categorized as follows: real emergency, intubation required without any delay; relative emergency, intubation required within $1 \mathrm{~h}$; deferred emergency, intubation required in more than $1 \mathrm{~h}$

Data are number $(\%)$ or mean $\pm \mathrm{SD}$

No significant differences were observed

whom intubation was guided by the protocol (Tables 1,5 ), the recommended individual procedures were satisfactorily applied in $75 \%$ of the overall cases $(866 / 1,152)$. There 
Table 5 Processes of medical care for the 244 evaluated procedures

\begin{tabular}{|c|c|c|c|}
\hline & $\begin{array}{l}\text { Control } \\
(n=121)\end{array}$ & $\begin{array}{l}\text { Intervention } \\
(n=123)\end{array}$ & $p$ \\
\hline \multicolumn{4}{|l|}{ Pre-procedure } \\
\hline 1. Presence of two operators & $79 / 121(65)$ & $123 / 123(100)$ & $<0.001$ \\
\hline 3. Preparation of long-term sedation & NR & $98 / 123(80)$ & NA \\
\hline 2. Fluid loading & $56 / 115(49)$ & $86 / 115(75)$ & $<0.001$ \\
\hline 4. Preoxygenation with NIPPV & $34 / 73(47)$ & $65 / 80(82)$ & $<0.001$ \\
\hline \multicolumn{4}{|l|}{ During procedure } \\
\hline \multirow{2}{*}{\multicolumn{4}{|c|}{$\begin{array}{l}\text { 5. Anesthetic drugs } \\
\text { Hypnotics }\end{array}$}} \\
\hline & & & \\
\hline Etomidate & $42 / 121(35)$ & 72/123 (58) & $<0.001$ \\
\hline Ketamine & $0 / 121(0)$ & 22/123 (18) & $<0.001$ \\
\hline Others hypnotics & $75 / 121(62)$ & $29 / 123(24)$ & $<0.001$ \\
\hline \multicolumn{4}{|l|}{ Muscle relaxants } \\
\hline Succinylcholine & $36 / 115(32)$ & $89 / 114(78)$ & $<0.001$ \\
\hline Other muscle relaxant & $42 / 121(35)$ & $9 / 123(7)$ & $<0.001$ \\
\hline 6. Sellick maneuver & $41 / 121(34)$ & $88 / 123(72)$ & $<0.001$ \\
\hline \multicolumn{4}{|l|}{ Post-procedure } \\
\hline 7. Capnography use & $0 / 121(0)$ & $69 / 123(56)$ & $<0.001$ \\
\hline 8. Early vasopressives drugs & NR & $14 / 19(77)$ & NA \\
\hline 9. Initiation of long-term sedation & NR & $81 / 123(65)$ & NA \\
\hline 10. Initial "protective ventilation" & NR & $74 / 105(70)$ & NA \\
\hline
\end{tabular}

The number for the denominator served to calculate the frequency (one case), spinal muscular amyotrophy (one case), acute renal of succinylcholine use taking into account only the cases without contraindications for succinylcholine use in both groups. Control: six contraindications to succinylcholine, no details have been recorded. Intervention: nine contraindications to succinylcholine: amyotrophic lateral sclerosis (one case), Guillain-Barre syndrome (one case), acute hyperkaliemia (one case), extreme bradycardia (one case) and anticipated difficult intubation (three cases) Data are number $(\%)$ or mean $\pm \mathrm{SD}$ $N R$ not recorded, $N A$ not applicable

were significant differences between the two phases for six of the ten processes, i.e., all that were recorded during the control phase (Table 5). Mean pressure support level delivered during the NIPPV for preoxygenation was similar for the two phases $\left(10 \pm 4 \mathrm{cmH}_{2} \mathrm{O}\right)$.

\section{Intubation-related complications}

The intubation procedure in the intervention phase $(n=121)$ was associated both with significantly lower life-threatening complications (21 vs. $34 \%, p=0.03$ ) (Fig. 1) and mild to moderate complications (9 vs. $21 \%$, $p=0.01$ ) (Fig. 2) than those in the control phase $(n=123)$. Severe hypoxemia and cardiovascular collapse, which were the main life-threatening complications after intubation, were reduced by half in the intervention group compared to the control group (Fig. 1).

We reported 12 esophageal intubations (6 in each group); 3 of them were complicated by severe hypoxemia (all in the control group, without capnography). Among the four esophageal intubations diagnosed by capnography, none of the patients developed severe hypoxemia, but two resulted in hemodynamic collapse. We did not find any significant change in the rate of complications, whether capnography was used or not. Difficult intubation was not different between the control and the intervention groups.

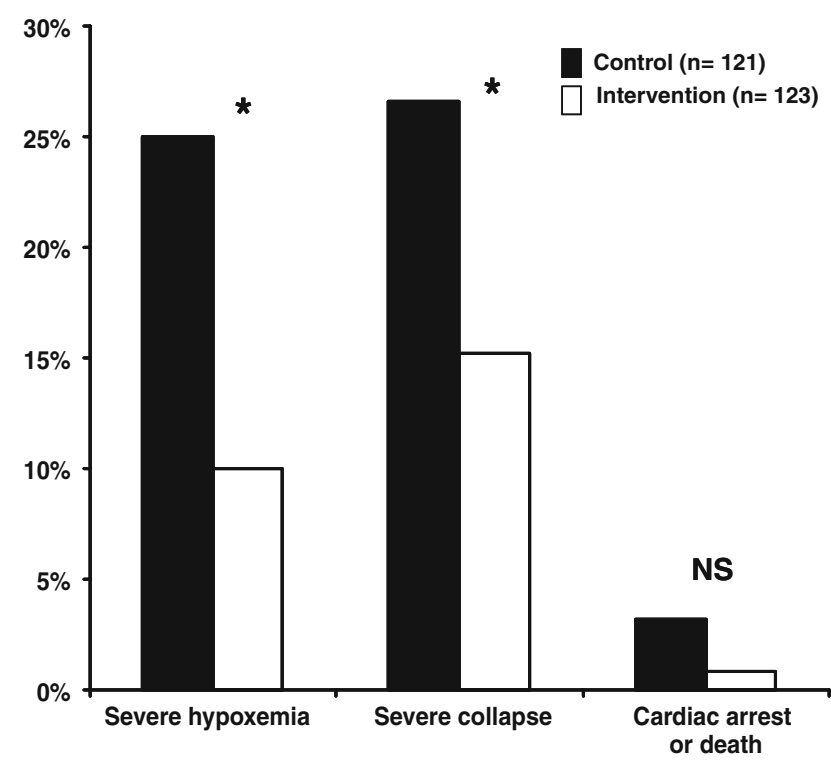

Fig. 1 Life-threatening complications occurring after all intubations performed during the control $(n=121)$ and the intervention $(n=123)$ phases. $* p<0.05$ versus control phase. NS not significant

Patient outcomes

There was no difference in length of mechanical ventilation [7 (2-14) vs. 8 (4-15) days, $p=0.65]$, ICU 


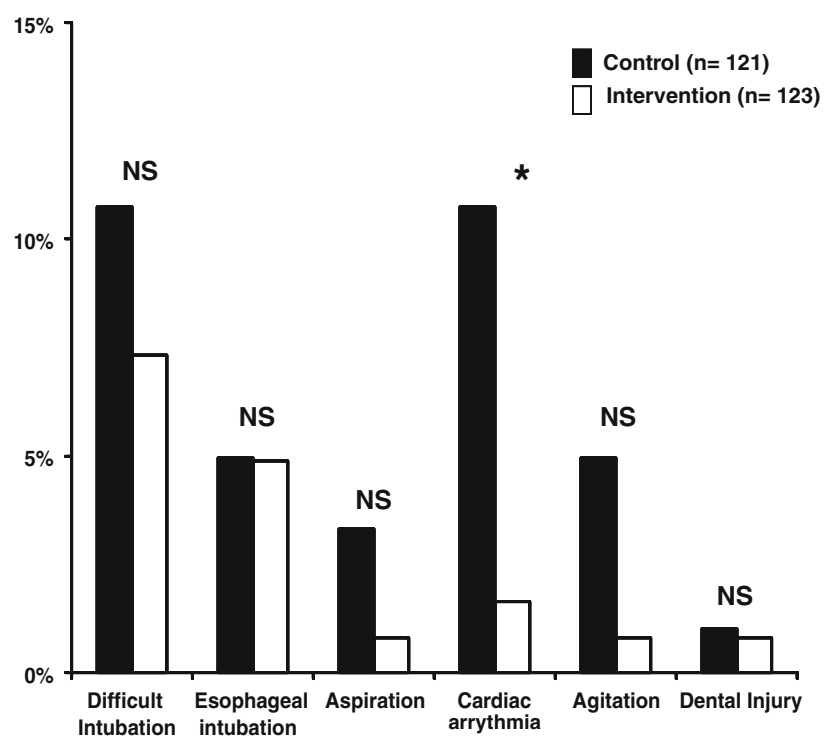

Fig. 2 Mild to moderate complications occurring after all intubations performed during the control $(n=121)$ and the intervention $(n=123)$ phases. $* p<0.05$ versus control phase. $N S$ not significant

ventilator-free days $[5 \quad(1-17)$ vs. 5 (1-17) days, $p=0.34]$, length of ICU stay [17 (6-31) vs. 22 (834) days, $p=0.35$ ] or ICU mortality (39 vs. $35 \%$, $p=0.42$ ) between control and intervention groups.

\section{Discussion}

Our results show that the implementation of an endotracheal intubation care bundle management in the ICU may reduce the incidence of life-threatening complications occurring within the first hour after intubation from 34 to $21 \%$. Severe hypoxemia and cardiovascular collapse incidences, which were the main life-threatening complications after intubation, were reduced by half in the intervention group compared to the control group.

This is the first study evaluating an intubation management protocol in the ICU that focuses on early complications after the procedure. The use of a checklist/ care bundles to improve patient safety is in keeping with current trends in anesthetic and intensive care medicine. Standardization of evidence-based practices in the care of critically ill patients has become accepted as a means of improving ICU care in many areas, such as out-of-hospital respiratory distress [15], analgesia and anesthesia $[16,23]$, weaning from mechanical ventilation $[16,17]$, sepsis management [18, 19] and surgical procedures [20]. By standardizing care, clinicians ensure that necessary procedures and therapies are carried out in a timely manner, especially in emergent situations. They also allow new practice changes to be more accurately monitored in terms of their effect on patient outcomes. Intubation procedures are often performed in the ICU and are associated with a high incidence of complications [1, $2,5,8]$. To date, the four observational studies that evaluated the severe complications related to intubation procedures reported rates ranging from 25 to $39 \%$ [2, 5, 6, 8]. This high incidence of complications led us to develop a local care bundle so as to improve the safety of ICU intubation procedure. However, the intubation procedure should include not only airway management, but also hemodynamic, gas exchange and neurologic care, which are often crucial in critically ill patients. Therefore, our care bundle added specific procedures, as a function of ICU patient organ failures (Table 1), to the usual upper airway management procedures. The protocol included several safety procedures divided into three periods (pre-, during and post-intubation) (Table 1). Six of the ten recommendations, NIPPV [1, 14], presence of two operators [2, 7], RSI (drugs and Sellick maneuver) [21], capnography [22] and protective ventilation [24], have been shown individually to improve critical and/or anesthesia care. The other four recommendations were developed on the basis of our clinical experience.

Preoxygenation is strongly recommended before intubation in the operating room, and its importance may be more pronounced in the ICU where patients often present oxygen transport limitations and are likely to require time-consuming airway management. Indeed, Mort [25] demonstrated that after conventional high-flow bag-valve mask preoxygenation of 4 min, patients with healthy lungs intubated for planned surgery increased their $\mathrm{PaO}_{2}$ from 79 to $404 \mathrm{mmHg}$, whereas for hypoxemic ICU patients intubated for acute respiratory failure the $\mathrm{PaO}_{2}$ increased from 64 to $87 \mathrm{mmHg}$. Recently, the same authors [3] reported that extending the preoxygenation period from 4 to 6 or $8 \mathrm{~min}$ is of limited benefit in improving oxygen tension in the majority of critical care patients, and they also found that nearly $50 \%$ of them presented severe desaturation during the intubation procedure. In hypoxemic patients, NIPPV improves oxygenation by delivering a high oxygen concentration, unloading respiratory muscle, recruiting alveoli and increasing lung volumes [25-27]. We recently showed that 3-min preoxygenation with NIPPV in acute respiratory failure patients before intubation in ICU limits the risk of acute hypoxemia during the procedure and improves $\mathrm{PaO}_{2}$ up to 30 min after intubation [1]. In the intervention group in which $82 \%$ of acute respiratory failure patients were preoxygenated with NIPPV, the incidence of post-intubation severe hypoxemia was $10 \%$, compared with $25 \%$ in the control group, in which only $47 \%$ of patients received NIPPV preoxygenation (Table 5; Fig. 1). We speculate that NIPPV was largely responsible for the improvement in the respiratory status of the intervention group. We recommended that two 
operators be present for the intubation procedure, because in a previous study [2] the presence of two operators (junior and senior) was an independent protective factor for intubation-related complications. In the present study, all intubations in the interventional group were performed in presence of two operators, compared with only $65 \%$ in the control group. In other words, a second pair of hands is often useful to manage intubation in a critically ill patient.

Airway management complications are more frequently encountered in the ICU than for scheduled surgery in the operating room [2, 4, 8]. After endotracheal intubation, it is of vital importance to confirm the endotracheal position of the tube. Although capnography is widely used and strictly recommended in the operating room, few studies have evaluated its impact in the ICU. In contrast to the control group in which capnography was never performed, $55 \%$ of procedures were verified by capnography in the intervention group (Table 5). In the remaining procedures, it could not be performed for logistical reasons or was forgotten by the team involved in the procedure. Among the 12 esophageal intubations (6 in each group), 4 were detected by the absence of an expiratory $\mathrm{CO}_{2}$ curve on the capnogram (all in the intervention group), and 8 were clinically detected. We did not find any significant change in the rate of complications whether capnography was used or not. Indeed, although the impact of capnography has been clearly demonstrated in pre-hospital care and anesthesia [4, 28], we could not reproduce these results, probably due to the lack of power of the present study.

Cardiovascular collapse frequently occurs within a few minutes following intubation [2, 13]. The additive effects of hypovolemia, the suppression of the endogenous activation of sympathic response by the anesthetics drugs $[22,29]$ as well as the intrathoracic positive pressure due to mechanical ventilation are implicated in cardiovascular collapse after intubation in critically ill patients. The impact of fluid loading to prevent cardiovascular collapse after ICU intubation has never been specifically studied, but we speculate that it could limit its occurrence, except in the case of cardiogenic edema. In the intervention group, in which $75 \%$ of patients received fluid loading, the incidence of severe post-intubation cardiovascular collapse was $15 \%$, in contrast to an incidence of $26 \%$ in the control group, in which only $49 \%$ received fluid loading (Table 5; Fig. 1). We speculate that fluid loading played a key role in the improvement of hemodynamic status in the intervention group. In the intervention group, we also observed a decrease in the incidence of arrhythmias, perhaps because of the combined effect of improved oxygenation with NIPPV and the beneficial effect of fluid loading on arterial blood pressure prior to anesthesia.

Administration of drugs should take into account the hemodynamic and neurologic status of patients and should have specific pharmokinetic properties for induction of anesthesia and long-term sedation. To limit the effects of drugs on cardiovascular status, we avoided agents such as propofol or thiopental [22] and proposed a rapid sequence induction with ketamine or etomidate and succinylcholine. Ketamine and etomidate are anesthetic agents widely used in pre-hospital or emergency room conditions because they have a rapid onset, a short halflife, are well tolerated hemodynamically and improve intubation conditions [12, 22]. Succinylcholine is a rapid onset, short-term muscle relaxant agent recommended for RSI, but its use should be avoided in some cases (Table 1) $[12,22]$. In the intervention group, $71 \%$ of intubations were performed with RSI compared to $34 \%$ in the control group $(p<0.0001)$ (Table 4$)$. To our knowledge, there is no published study testing the safety and efficacy of muscle relaxants for urgent intubation of hospitalized patients. However, Schmidt et al. [7] reported that attending physicians tended to use muscle relaxants significantly more frequently than juniors, and muscle relaxants were used more frequently in groups with fewer complications.

In the intervention group, the use of propofol decreased and use of ketamine and etomidate significantly increased (Table 4). We speculate that the increased use of etomidate and ketamine may have also contributed to the improvement of the hemodynamic status in the intervention group. Etomidate is an agent suspected to have a negative effect on adrenal function [30]; however, these results are still controversial [31]. Furthermore, the adrenal consequences of etomidate have never been compared to its cardiovascular safety and its properties to improve intubation conditions in critically ill patients [12, 22]. Nonetheless, we proposed ketamine use, which recently was reported to be equivalent to etomidate in terms of outcome in pre-hospital care [32], as an alternative hypnotic agent. To avoid agitation of critically ill patients, which is known to have deleterious consequences on ICU patient morbidity [16, 33], we started sedation immediately after intubation in the intervention group in $65 \%$ of the procedures, whereas its use was not recorded in the control group (Table 4). Because of the low incidence and thus a low power, we could not report any significant difference in agitation between the two groups.

Once a patient is intubated and sedated, the initial ventilatory settings should be protective using limited tidal volume, because high tidal volume $(>12 \mathrm{ml} / \mathrm{kg})$ and high intrathoracic pressures may worsen re-ventilation collapse in unstable ICU patients.

Our study has several limitations. First, the design of our non-randomized study does not enable us to definitely demonstrate a causality link. This design, involving a comparison of pre-intervention data (control group) with post-intervention data (intervention group) and the consecutive recruitment of the two groups of patients from 
the same ICUs at the same hospitals, was chosen because it was not possible to randomly assign the use of the intubation management protocol to specific units without significant cross-contamination. One danger of this design is confounding by secular trends. We therefore confined the duration of the study to less than 6 months, since a change in others care procedures and outcomes of the observed magnitude are unlikely to occur in such a short period as a result of secular trends alone. Second, the design does not permit us to determine which recommendation was especially useful for the observed decreased in immediate severe life-threatening complications subsequent to intubation. We established a global program for the improvement of intubation management and then assessed the impact of all the guidelines. The lack of recording of four of the ten recommendations during the control period (Table 5) and the limited number of studied intubation procedures did not allow us to perform relevant multivariable analysis. More than to study the effect of one specific intervention, the aim of our trial was to evaluate the benefit of an overall care bundle in the context of emergency intubation in ICU. Despite these limitations, our study supports the importance of achieving targeted end points for the management of emergency intubation in the ICU so as to decrease related complications.

This type of study design (quality control observation phase followed by an intervention phase) is adequate in order to improve clinical practices. Moreover, most of the studies that investigated the impact of guideline implementation [15-17, 19], including several recommendations, used a "before-after" design. Indeed, some processes may provide physiological benefit when applied alone, but cannot be shown to improve clinical outcome. On the other hand, a group of evidence-based treatments related to a disease process, instituted rapidly and together over a specific timeframe, is frequently associated with better outcomes than when they are executed individually. Because of being a bundle care study of ten recommendations, the overall bundle compliance did not reach $100 \%$, but it was ranged from 56 to $100 \%$ according the process-of-care variables, which is very high compliance compared to others similar studies that reported ranges from 5 to $87 \%[15,17,18,34]$. These low rates of compliance showed that barriers to implementing bundles are relevant [34].

Finally, outcomes upon ICU discharge were not different between the two groups, but our study design was not adequate to evaluate outcomes beyond that of the procedure. Further studies are needed to clarify the impact of such a bundle on outcomes [35].

In conclusion, we showed that a ten-point care bundle management of intubation in the ICU, including fluid loading, preoxygenation with non invasive positive pressure ventilation, rapid sequence induction and capnography to check the endotracheal tube position, decreased the rate of severe hypoxemia and hemodynamic collapse occurring within the firsst hour following intubation. Future studies should, however, investigate whether a decrease in immediate life-threatening complications can lead to the improvement of outcomes upon ICU discharge.

Acknowledgments The authors wish to thank all the personnel of the intensive care units who participated in the implementation of these intubation guidelines. They are grateful to Patrick McSweeny for his English editing and Emmanuel Marret for statistical help.

Conflict of interest statement Support was provided solely from institutional and/or departmental sources.

\section{References}

1. Baillard C, Fosse JP, Sebbane M, Chanques G, Vincent F, Courouble P, Cohen Y, Eledjam JJ, Adnet F, Jaber S (2006) Noninvasive ventilation improves preoxygenation before intubation of hypoxic patients. Am J Respir Crit Care Med 174:171-177

2. Jaber S, Amraoui J, Lefrant JY, Arich C, Cohendy R, Landreau L, Calvet Y, Capdevila X, Mahamat A, Eledjam JJ (2006) Clinical practice and risk factors for immediate complications of endotracheal intubation in the intensive care unit: a prospective, multiple-center study. Crit Care Med 34:2355-2361

3. Mort TC, Waberski BH, Clive J (2009) Extending the preoxygenation period from 4 to 8 mins in critically ill patients undergoing emergency intubation. Crit Care Med 37:68-71
4. Petrini F, Accorsi A, Adrario E, Agro F Amicucci G, Antonelli M, Azzeri F, Baroncini S, Bettelli G, Cafaggi C, Cattano D, Chinelli E, Corbanese U, Corso R, Della Puppa A, Di Filippo A, Facco E, Favaro R, Favero R, Frova G Giunta F, Giurati G, Giusti F, Guarino A, Iannuzzi E, Ivani G, Mazzon D, Menarini M, Merli G, Mondello E, Muttini S, Nardi G, Pigna A, Pittoni G, Ripamonti D, Rosa G, Rosi R, Salvo I, Sarti A, Serafini G, Servadio G, Sgandurra A, Sorbello M, Tana F, Tufano R, Vesconi S, Villani A, Zauli M (2005) Recommendations for airway control and difficult airway management. Minerva Anestesiol 71:617-657
5. Griesdale DE, Bosma TL, Kurth T, Isac G, Chittock DR (2008) Complications of endotracheal intubation in the critically ill. Intensive Care Med 34:1835-1842

6. Le Tacon S, Wolter P, Rusterholtz T, Harlay M, Gayol S, Sauder P, Jaeger A (2000) Complications of difficult tracheal intubations in a critical care unit. Ann Fr Anesth Réanim 19:719724

7. Schmidt UH, Kumwilaisak K, Bittner E, George E, Hess D (2008) Effects of supervision by attending anesthesiologists on complications of emergency tracheal intubation. Anesthesiology 109:973-977 
8. Schwartz DE, Matthay MA, Cohen NH (1995) Death and other complications of emergency airway management in critically ill adults. Anesthesiology 82:367-376

9. (2003) Practice guidelines for management of the difficult airway: an updated report by the American Society of Anesthesiologists Task Force on Management of the Difficult Airway. Anesthesiology 98:1269-1277

10. Adnet F, Jouriles NJ, Le Toumelin P, Hennequin B, Taillandier C, Rayeh F Couvreur J, Nougiere B, Nadiras P, Ladka A, Fleury M (1998) Survey of out-of-hospital emergency intubations in the French prehospital medical system: a multicenter study. Ann Emerg Med 32:454-460

11. Girault C, Auriant I, Jaber S (2008) Safety practices procedures for mechanical ventilation. Ann Fr Anesth Reanim 27:e77-e89

12. Smith DC, Bergen JM, Smithline H, Kirschner R (2000) A trial of etomidate for rapid sequence intubation in the emergency department. J Emerg Med 18:13-16

13. Leibowitz AB (2006) Tracheal intubation in the intensive care unit: extremely hazardous even in the best of hands. Crit Care Med 34:2497-2498

14. Delay JM, Sebbane M, Jung B, Nocca D, Verzilli D, Pouzeratte Y, Kamel ME Fabre JM, Eledjam JJ, Jaber S (2008) The effectiveness of noninvasive positive pressure ventilation to enhance preoxygenation in morbidly obese patients: a randomized controlled study. Anesth Analg 107:1707-1713

15. Stiell IG, Spaite DW, Field B, Nesbitt LP, Munkley D, Maloney J, Dreyer J, Toohey LL, Campeau T, Dagnone E, Lyver M, Wells GA (2007) Advanced life support for out-of-hospital respiratory distress. N Engl J Med 356:2156-2164

16. Chanques G, Jaber S, Barbotte E, Violet S, Sebbane M, Perrigault PF, Mann C, Lefrant JY, Eledjam JJ (2006) Impact of systematic evaluation of pain and agitation in an intensive care unit. Crit Care Med 34:1691-1699

17. Girard TD, Kress JP, Fuchs BD, Thomason JW, Schweickert WD, Pun BT, Taichman DB, Dunn JG, Pohlman AS, Kinniry PA, Jackson JC, Canonico AE, Light RW, Shintani AK, Thompson JL, Gordon SM, Hall JB, Dittus RS, Bernard GR, Ely EW (2008) Efficacy and safety of a paired sedation and ventilator weaning protocol for mechanically ventilated patients in intensive care (Awakening and Breathing Controlled trial): a randomised controlled trial. Lancet 371:126-134
18. Ferrer R, Artigas A, Levy MM, Blanco J, Gonzalez-Diaz G, Garnacho-Montero J, Ibanez J, Palencia E, Quintana M, de la Torre-Prados MV (2008) Improvement in process of care and outcome after a multicenter severe sepsis educational program in Spain. JAMA 299:2294-2303

19. Micek ST, Roubinian N, Heuring T, Bode M, Williams J, Harrison C, Murphy T, Prentice D, Ruoff BE Kollef MH (2006) Before-after study of a standardized hospital order set for the management of septic shock. Crit Care Med 34:2707-2713

20. Haynes AB, Weiser TG, Berry WR, Lipsitz SR, Breizat AH, Dellinger EP, Herbosa T, Joseph S, Kibatala PL, Lapitan MC, Merry AF, Moorthy K, Reznick RK, Taylor B, Gawande AA (2009) A surgical safety checklist to reduce morbidity and mortality in a global population. N Engl J Med 360:491-499

21. Reynolds SF, Heffner J (2005) Airway management of the critically ill patient: rapid-sequence intubation. Chest 127:1397-1412

22. Walz JM, Zayaruzny M, Heard SO (2007) Airway management in critical illness. Chest 131:608-620

23. Brattebo G, Hofoss D, Flaatten H, Muri AK, Gjerde S, Plsek PE (2002) Effect of a scoring system and protocol for sedation on duration of patients' need for ventilator support in a surgical intensive care unit. BMJ 324:13861389

24. Schultz MJ, Haitsma JJ, Slutsky AS, Gajic O (2007) What tidal volumes should be used in patients without acute lung injury? Anesthesiology 106:12261231

25. Mort TC (2005) Preoxygenation in critically ill patients requiring emergency tracheal intubation. Crit Care Med 33:2672-2675

26. Jaber S, Delay JM, Chanques G, Sebbane M, Jacquet E, Souche B, Perrigault PF, Eledjam JJ (2005) Outcomes of patients with acute respiratory failure after abdominal surgery treated with noninvasive positive pressure ventilation. Chest 128:2688-2695

27. Jaber S, Fodil R, Carlucci A, Boussarsar M, Pigeot J, Lemaire F, Harf A, Lofaso F, Isabey D, Brochard L (2000) Noninvasive ventilation with heliumoxygen in acute exacerbations of chronic obstructive pulmonary disease. Am J Respir Crit Care Med 161:11911200
28. Donald MJ, Paterson B (2006) End tidal carbon dioxide monitoring in prehospital and retrieval medicine: a review. Emerg Med J 23:728-730

29. Walz JM, Heard SO (2005) Making tracheal intubation safer in the critically ill patient. Crit Care Med 33:2716-2717

30. Sprung CL, Annane D, Keh D, Moreno R, Singer M, Freivogel K, Weiss YG, Benbenishty J, Kalenka A, Forst H, Laterre PF, Reinhart K, Cuthbertson BH, Payen D, Briegel J (2008) Hydrocortisone therapy for patients with septic shock. N Engl J Med 358:111-124

31. Kamp R, Kress JP (2007) Etomidate, sepsis, and adrenal function: not as bad as we thought? Crit Care 11:145

32. Jabre P, Combes X, Lapostolle F, Dhaouadi M, Ricard-Hibon A, Vivien B, Bertrand L, Beltramini A, Gamand P, Albizzati S, Perdrizet D, Lebail G, Chollet-Xemard C, Maxime V, BrunBuisson C, Lefrant JY, Bollaert PE, Megarbane B, Ricard JD, Anguel N, Vicaut E, Adnet F (2009) Etomidate versus ketamine for rapid sequence intubation in acutely ill patients: a multicentre randomised controlled trial. Lancet

33. Jaber $\mathrm{S}$, Chanques $\mathrm{G}$, Altairac $\mathrm{C}$, Sebbane M, Vergne C, Perrigault PF, Eledjam JJ (2005) A prospective study of agitation in a medical-surgical ICU: incidence, risk factors, and outcomes. Chest 128:2749-2757

34. Jaeschke R, Jankowski M, Brozek J, Antonelli M (2009) How to develop guidelines for clinical practice. Minerva Anestesiol 75:504-508

35. Baillard C, Jaber S (2007) NCT00472160 CT. Preoxygenation using NIV in hypoxemic patients (preoxy study)

36. Le Gall JR, Lemeshow S, Saulnier F (1993) A new Simplified Acute Physiology Score (SAPS II) based on a European/North American multicenter study. JAMA 270:2957-2963 